\title{
Basic science (July 2006)
}

1. Ai LB, Tao QA, Zhong S, Fields CR, Kim WJ, Lee MW, Cui Y, Brown KD, Robertson KD. Inactivation of Wnt inhibitory factor-1 (WIF1) expression by epigenetic silencing is a common event in breast cancer [Abstract]. Carcinogenesis 2006; 27: 1341-1348.

2. Allan AL, George R, Vantyghem SA, Lee MW, Hodgson NC, Engel CJ, Holliday RL, Girvan DP, Scott LA, Postenka CO, Al-Katib W, Stitt LW, Uede T, Chambers AF, Tuck AB. Role of the integrin-binding protein osteopontin in lymphatic metastasis of breast cancer [Abstract]. $A m ~ J$ Pathol 2006; 169: 233-246.

3. Allen-Brady K, Cannon-Albright LA, Neuhausen SL, Camp NJ. A role for XRCC4 in age at diagnosis and breast cancer risk [Abstract]. Cancer Epidem Biomark Prevent 2006; 15: 1306-1310.

4. Benakanakere I, Besch-Williford C, Schnell J, Brandt S, Ellersieck MR, Molinolo A, Hyder SM. Natural and synthetic progestins accelerate 7,12-dimethylbenz[a] anthracene-initiated mammary tumors and increase angiogenesis in Sprague-Dawley rats [Abstract]. Clin Cancer Res 2006; 12: 4062-4071.

5. Boersma BJ, Howe TM, Goodman JE, Yfantis HG, Lee DH, Chanock SJ, Ambs S. Association of breast cancer outcome with status of p53 and MDM2 SNP309 [Abstract]. J Natl Cancer Inst 2006; 98: 911-919.

6. Burrows C, Holly JMP, Laurence NJ, Vernon EG, Carter JV, Clark MA, McIntosh J, McCaig C, Winters ZE, Perks CM. Insulin-like growth factor binding protein 3 has opposing actions on malignant and nonmalignant breast epithelial cells that are each reversible and dependent upon cholesterol-stabilized integrin receptor complexes [Abstract]. Endocrinology 2006; 147: 3484-3500.
7. Caffarel MM, Sarrio D, Palacios J, Guzman M, Sanchez C. Delta(9)-tetrahydrocannabinol inhibits cell cycle progression in human breast cancer cells through Cdc2 regulation [Abstract]. Cancer Res 2006; 66: 6615-6621.

8. Cazzaniga M, Severi G, Casadio C, Chiapparini L, Veronesi U, Decensi A. Atypia and Ki-67 expression from ductal lavage in women at different risk for breast cancer [Abstract]. Cancer Epidem Biomark Prevent 2006; 15: 1311-1315.

9. Chandanos E, Lindblad M, Jia C, Rubio CA, Ye W, Lagergren J. Tamoxifen exposure and risk of oesophageal and gastric adenocarcinoma: a population-based cohort study of breast cancer patients in Sweden [Abstract]. Brit J Cancer 2006; 95: 118-122.

10. Cross SS, Harrison RF, Balasubramanian SP, Lippitt JM, Evans CA, Reed MWR, Holen I. Expression of receptor activator of nuclear factor kappa beta ligand (RANKL) and tumour necrosis factor related, apoptosis inducing ligand (TRAIL) in breast cancer, and their relations with osteoprotegerin, oestrogen receptor, and clinicopathological variables [Abstract]. J Clin Pathol 2006; 59: 716-720.

11. Dahl E, Kristiansen G, Gottlob K, Klaman I, Ebner E, Hinzmann B, Hermann K, Pilarsky C, Durst $M$, Klinkhammer-Schalke $M$, Blaszyk $H$, Knuechel R, Hartmann A, Rosenthal A, Wild PJ. Molecular profiling of laser-microdissected matched tumor and normal breast tissue identifies karyopherin alpha 2 as a potential novel prognostic marker in breast cancer [Abstract]. Clin Cancer Res 2006; 12: 3950-3960.

12. Dong J, Tsai-Morris CH, Dufau ML. A novel estradiol/estrogen receptor alpha-dependent transcriptional mechanism controls expression of the human prolactin receptor [Abstract]. J Biol Chem 2006; 281: 18825-18836.

13. Ellis MJ, Too $Y$, Young $O$, White $S$, Proia $A D$, Murray J, Renshaw L, Faratian D, Thomas J, Dowsett M, Krause A, Evans DB, Miller WR, Dixon JM. Estrogen-independent proliferation is 
present in estrogen-receptor HER2-positive primary breast cancer after neoadjuvant letrozole [Abstract]. J Clin Oncol 2006; 24: 3019-3025.

14. Fournier MV, Martin KJ, Kenny PA, Xhaja K, Bosch I, Yaswen P, Bissell MJ. Gene expression signature in organized and growth-arrested mammary acini predicts good outcome in breast cancer [Abstract]. Cancer Res 2006; 66: 7095-7102.

15. Francini G, Petrioli R, Montagnani A, Cadirni A, Campagna S, Francini E, Gonnelli S. Exemestane after tamoxifen as adjuvant hormonal therapy in postmenopausal women with breast cancer: effects on body composition and lipids [Abstract]. Bri J Cancer 2006; 95: 153-158.

16. Frasor J, Chang EC, Komm B, Lin CY, Vega VB, Liu ET, Miller LD, Smeds J, Bergh J, Katzenellenbogen BS. Gene expression preferentially regulated by tamoxifen in breast cancer cells and correlations with clinical outcome [Abstract]. Cancer Res 2006; 66: 7334-7340.

17. Furuta S, Wang JM, Wei SZ, Jeng YM, Jiang XZ, Gu BN, Chen PL, Lee E, Lee WH. Removal of BRCA1/CtIP/ZBRK1 repressor complex on ANG1 promoter leads to accelerated mammary tumor growth contributed by prominent vasculature [Abstract]. Cancer Cell 2006; 10: 13-24.

18. Gonzalez L, Agullo-Ortuno MT, GarciaMartinez JM, Calcabrini A, Gamallo C, Palacios J, Aranda A, Martin-Perez J. Role of c-Src in human MCF7 breast cancer cell tumorigenesis [Abstract]. J Biol Chem 2006; 281: 20851-20864.

19. Huang Y, Keen JC, Pledgie A, Marton LJ, Zhu T, Sukumar S, Park BH, Blair B, Brenner K, Casero RA, Davidson NE. Polyamine analogues down-regulate estrogen receptor alpha expression in human breast cancer cells [Abstract]. J Biol Chem 281: 19055-19063.

20. Jerome L, Alami N, Belanger S, Page V, Yu QN, Paterson J, Shiry L, Pegram M, Leyland-Jones B. Recombinant human insulin-like growth factor binding protein 3 inhibits growth of human epidermal growth factor receptor-2-overexpressing breast tumors and potentiates Herceptin activity in vivo [Abstract]. Cancer Res 2006; 66: 7245-7252.

21. Kawakubo H, Brachtel E, Hayashida T, Yeo G, Kish J, Muzikansky A, Walden PD, Maheswaran S. Loss of B-cell translocation gene-2 in estrogen receptor-positive breast carcinoma is associated with tumor grade and overexpression of cyclin D1 protein [Abstract]. Cancer Res 2006; 66: 7075-7082.

22. Kote-Jarai Z, Matthews L, Osorio A, Shanley S, Giddings I, Moreews F, Locke I, Evans DG, Eccles DGACCC. Accurate prediction of BRCA1 and
BRCA2 heterozygous genotype using expression profiling after induced DNA damage [Abstract]. Clin Cancer Res 2006; 12: 3896-3901.

23. Kumar SR, Singh J, Xia GB, Krasnoperov V, Hassanieh L, Ley EJ, Scehnet J, Kumar NG, Hawes D, Press MF, Weaver FA, Gill PS. Receptor tyrosine kinase EphB4 is a survival factor in breast cancer [Abstract]. Am J Pathol 2006; 169: 279-293.

24. Larson PS, de las Morenas A, Cerda SR, Bennett SR, Cupples LA, Rosenberg CL. Quantitative analysis of allele imbalance supports atypical ductal hyperplasia lesions as direct breast cancer precursors [Abstract]. J Pathol 2006; 209: 307-316.

25. Lau QC, Raja E, Salto-Terez M, Liu Q, Ito K, Inoue M, Putti TC, Loh M, Ko TK, Huang $\mathrm{CH}$, Bhalla KN, Zhu T, Ito Y, Sukumar S. RUNX3 is frequently inactivated by dual mechanisms of protein mislocalization and promoter hypermethylation in breast cancer [Abstract]. Cancer Res 2006; 66: 6512-6520.

26. Locke I, Kote-Jarai Z, Bancroft E, Bullock S, Jugurnauth $S$, Osin $P$, Nerurkar A, Izatt L, Pichert G, Gui GPH, Eeles RA. Loss of heterozygosity at the BRCA1 and BRCA2 loci detected in ductal lavage fluid from BRCA gene mutation carriers and controls [Abstract]. Cancer Epidemiol Biomar Prevent 2006; 15: 1399-1402.

27. Mitra SK, Lim ST, Chi A, Schlaepfer DD. Intrinsic focal adhesion kinase activity controls orthotopic breast carcinoma metastasis via the regulation of urokinase plasminogen activator expression in a syngeneic tumor model [Abstract]. Oncogene 2006; 25: 4429-4440.

28. Nam JS, Kang MJ, Suchar AM, Shimamura T, Kohn EA, Michalowska AM, Jordan VC, Hirohashi S, Wakefield LM. Chemokine (C-C motif) ligand 2 mediates the prometastatic effect of dysadherin in human breast cancer cells [Abstract]. Cancer Res 2006; 66: 7176-7184.

29. Oxelmark E, Roth JM, Brooks PC, Braunstein SE, Schneider RJ, Garabedian MJ. The cochaperone p23 differentially regulates estrogen receptor target genes and promotes tumor cell adhesion and invasion [Abstract]. $\mathrm{Mol}$ Cell Biol 2006; 26: 5205-5213.

30. Palomares MR, Machia JRB, Lehman CD, Daling JR, McTiernan A. Mammographic density correlation with gail model breast cancer risk estimates and component risk factors [Abstract]. Cancer Epidemiol Biomar Prevent 2006; 15: 1324-1330.

31. Peng XJ, Mehta R, Wang S, Chellappan S, Mehta RG. Prohibitin is a novel target gene of 
vitamin $\mathrm{D}$ involved in its antiproliferative action in breast cancer cells [Abstract]. Cancer Res 2006; 66: 7361-7369.

32. Reis JS, Savage K, Lambros MBK, James M, Steele D, Jones RL, Dowsett M. Cyclin D1 protein overexpression and CCND1 amplification in breast carcinomas: an immunohistochemical and chromogenic in situ hybridisation analysis [Abstract]. Modern Pathol 2006; 19: 999-1009.

33. Riggins RB, Thomas KS, Ta HQ, Wen J, Davis RJ, Schuh NR, Donelan SS, Owen KA, Gibson MA, Shupnik MA, Silva CM, Parsons SJ, Clarke R, Bouton AH. Physical and functional interactions between Cas and c-Src induce tamoxifen resistance of breast cancer cells through pathways involving epidermal growth factor receptor and signal transducer and activator of transcription 5b [Abstract]. Cancer Res 2006; 66: 7007-7015.

34. Ring BZ, Seitz RS, Beck R, Shasteen WJ, Tarr SM, Cheang MCU, Yoder BJ, Budd GT, Nielsen TO, Hicks DG, Estopinal NC, Ross DT. Novel prognostic immunohistochemical biomarker panel for estrogen receptor-positive breast cancer [Abstract]. J Clin Oncol 2006; 24: 3039-3047.

35. Rohan TE, Li SQ, Hartwick R, Kandel RA. p53 alterations and protein accumulation in benign breast tissue and breast cancer risk: A cohort study [Abstract]. Cancer Epidemiol Biomar Prevent 2006; 15: 1316-1323.

36. Schmitt M, Walker MP, Richards RG, Bocchinfuso WP, Fukuda T, Medina D, Kittrel FS, Korach KS, DiAugustine RP. Expression of heregulin by mouse mammary tumor cells: Role in activation of ErbB receptors [Abstract]. $\mathrm{Mol}$ Carcinogen 2006; 45: 490-505.

37. Shen Q, Zhang Y, Uray IP, Hill JL, Kim HT, Lu CH, Young MR, Gunther EJ, Hilsenbeck SG, Chodosh LA, Colburn NH, Brown PH. The AP-1 transcription factor regulates postnatal mammary gland development [Abstract]. Develop Biol 2006; 295: 589-603.

38. Shrivastava A, Tiwari M, Sinha RA, Kumar A, Balapure AK, Bajpai VK, Sharma R, Mitra K, Tandon A, Godbole MM. Molecular iodine induces caspase-independent apoptosis in human breast carcinoma cells involving the mitochondriamediated pathway [Abstract]. J Biol Chem 2006; 281: 19762-19771.

39. Shukla V, Coumoul X, Cao L, Wang RH, Xiao CY, Xu XL, Ando S, Yakar S, LeRoith D, Deng CX. Absence of the full-length breast cancerassociated gene- 1 leads to increased expression of insulin-like growth factor signaling axis members [Abstract]. Cancer Res 2006; 66: 7151-7157.
40. Shulewitz M, Soloviev I, Wu T, Koeppen H, Polakis P, Sakanaka C. Repressor roles for TCF-4 and Sfrp1 in Wnt signaling in breast cancer [Abstract]. Oncogene 2006; 25: 4361-4369.

41. Spruck C, Sun D, Fiegl H, Marth C, MuellerHolzner E, Goebel G, Widschwendter M, Reed SI. Detection of low molecular weight derivatives of cyclin E1 is a function of cyclin E1 protein levels in breast cancer [Abstract]. Cancer Res 2006; 66: 7355-7360.

42. Stacey SN, Sulem P, Johannsson OT, Helgason A, Gudmundsson J, Kostic JP, Kristjansson K, Jonsdottir T, Sigurdsson H, Hrafnkelsson J, Johannsson J, Sveinsson T, Myrdal G, Grimsson HN, Bergthorsson JT, Amundadottir LT, Gulcher JR, Thorsteinsdottir U, Kong A, Stefansson K. The BARD1 Cys557Ser variant and breast cancer risk in lceland - art. no. E217. Plos Med 2006; 3: 1103-1113.

43. van der Hout $A H$, van den Ouweland AMW, van der Luijt RB, Gille HJP, Bodmer D, Bruggenwirth $\mathrm{H}$, Mulder IM, van der Vlies $P$, Elfferich $P$, Huisman MT, ten Berge AM, Kromosoeto J, Jansen RPM, van Zon PHA, Vriesman T, Arts N, Lange MBD, Oosterwijk JC, Meijers-Heijboer H, Ausems M, Hoogerbrugge N, Verhoef S, Halley DJJ, Vos YJ, Hogervorst F, Ligtenberg M, Hofstra RMW. A DGGE system for comprehensive mutation screening of BRCA1 and BRCA2: Application in a Dutch cancer clinic setting [Abstract]. Human Mutat 2006; 27: 654-666.

44. Wang W, Epler J, Salazar LG, Riddell SR. Recognition of breast cancer cells by $\mathrm{CD} 8(+)$ cytotoxic T-cell clones specific for NY-BR-1 [Abstract]. Cancer Res 2006; 66: 6826-6833.

45. Woditschka S, Haag JD, Waller JL, Monson DM, Hitt AA, Brose HL, Hu R, Zheng Y, Watson PA, Kim K, Lindstrom MJ, Mau B, Steele VE, Lubet RA, Gould MN. Neu-induced retroviral rat mammary carcinogenesis: A novel chemoprevention model for both hormonally responsive and nonresponsive mammary carcinomas [Abstract]. Cancer Res 2006; 66: 6884-6891.

46. Yu XC, Fu SA, Lai MY, Baer R, Chen JJ. BRCA1 ubiquitinates its phosphorylation-dependent binding partners CtIP [Abstract]. Gene Develop 2006; 20: 1721-1726.

R. Sutherland, J. Scorer Cancer Research Program Garvan Institute of Medical Research Darlinghurst, NSW, Australia 